\title{
RINGS WITH INVOLUTION ALL OF WHOSE SYMMETRIC ELEMENTS ARE NILPOTENT OR REGULAR
}

\author{
J. CHACRON AND M. CHACRON ${ }^{1}$
}

\begin{abstract}
It is shown that noetherian rings with involution having all their symmetric elements nilpotent or regular are orders in artinian rings with involution having all their symmetric elements nilpotent or invertible.
\end{abstract}

For the terminology used in this paper see, for example, [1]. Rings with involution $R$ in which each nonzero symmetric element is invertible were first studied by Osborn [11]. Since several authors (see [3], [9], [12]) generalized Osborn's condition to rings $R$ all of whose symmetric elements are nilpotent or invertible (2) and since noetherian rings (with or without involution) in which each element is nilpotent or right regular were recently studied in [7], it is therefore legitimate to study, at least in the noetherian case, rings $R$ in which (1) each symmetric element is nilpotent or regular (or equivalently nilpotent or right regular). In this paper we prove

THEOREM. Let $R$ be any ring with involution satisfying (1). If $R$ is a nonnil noetherian ring then $R$ is an order in an artinian ring with unity $Q$ in which each symmetric element is nilpotent or invertible.

The Theorem generalizes Lesieur's results [7, Theorem 4] and yields the following generalization of Lanski's results:

COROLlaRY. If $R$ is as in the Theorem, then the nilpotent radical $N$ of $R$ contains any nilpotent symmetric element of the form $x x^{*}$ or $x+x^{*}$ and the factor ring $R / N$ is one of the following types: (i) a skew-domain; (ii) a subdirect product of two isomorphic sken-domains; (iii) an order in the $2 \times 2$ matrices over a field.

Received by the editors January 13, 1972.

AMS (MOS) subject classifications (1970). Primary 16A28; Secondary 16A48.

Key words and phrases. Noetherian rings with involution, regular symmetric elements, nilpotent symmetric elements.

${ }^{1}$ This research was supported by N.R.C. Grant A7876 and by a summer fellowship of the Canadian Mathematical Congress (branch at Universite Laval).

(c) American Mathematical Society 1973 
One could wonder if any semiprime ring $R$ satisfying (1) with or without the noetherian assumption must be of one of the types in the Corollary. The following example, which is attributed to W. Martindale, shows that the dropping of the noetherian assumption requires counterpart.

EXAMPLE 1. Let $F$ be any field. Let $x, y$ be noncommuting indeterminates and let $\left(x^{2}\right)$ be the ideal generated by $x^{2}$ in the polynomial ring $F[x, y]$. One can turn the latter ring into a ring with involution * by declaring the scalars and the indeterminates to be symmetric elements. Since $\left(x^{2}\right)$ will be a symmetric ideal, that is, $\left(x^{2}\right)=\left(x^{2}\right)^{*}$, it follows that the factor ring $R=F[x, y] /\left(x^{2}\right)$ carries the induced involution $W(x, y)+\left(x^{2}\right) \rightarrow$ $W^{*}(x, y)+\left(x^{2}\right)$. Under this involution the ring $R$ is a semisimple ring satisfying (1). Since $R$ is a prime ring, type (ii) is excluded. Since $x^{2}=0$ on $R$, type (i) is also excluded. Finally type (iii) is ruled out by observing that $R$ is not a Goldie ring (and consequently $R$ cannot be an order in the $2 \times 2$-matrices over a field).

Now to the proofs of the Theorem and its Corollary. We begin with

PROPOSITION 1. Let $R$ be any ring with involution having all its symmetric elements nilpotent. Assume, further, at least one of the following conditions:

(i) $R$ is noetherian;

(ii) the symmetric elements of $R$ have a bounded index.

Then $R$ is a nil ring.

Proof. Since the nil radical $N$ of $R$ is a symmetric ideal such that $R$ and the factor ring $R / N$ satisfy at the same time the above conclusion and since conditions (i) and (ii) are inherited by the latter ring equipped with the induced involution $x+N \rightarrow x^{*}+N$, it suffices therefore to prove that if $N=0$ then $R=0$. First we show that $s=0$ for each symmetric element $s=s^{*}$ of $R$. Since each symmetric element of $R$ is nilpotent it suffices to prove this for each square-zero element $s=s^{*}$. Now for each $x \in R$, $d=s x+x^{*} s=d^{*}$ is nilpotent and

$$
d^{n} s=\left((s x)^{n}+\left(x^{*} s\right)^{n}+s y_{n} s\right) s=(s x)^{n} s,
$$

so there is an integer $n$ not exceeding the index of nilpotence of $d$ plus 1 such that $(s x)^{n} s=0$. From this $(s x)^{n+1}=0$. It follows that the right ideal $\mid s)$ generated by $s$ is nil. In case (i), one gets that either $R=0$ or $R$ contains a nonzero nilpotent ideal. In case (ii), Levitski's result [4, Lemma 1] yields the same conclusion. One must conclude that $s=0$. Then $x^{2}=0$ for each $x \in R$. By Levitski's result, $R=0$.

Proposition 1 answers K. McCrimmon's question [8, p. 142] in a special case. Along the lines of the proof of Lesieur's theorem let us now show 
Proposition 2. Let $R$ be any noetherian ring with involution satisfying (1). If $R$ is not nil (or, better, not nilpotent), then $R$ is an order in an artinian ring $Q$ with an involution extending the base involution.

Proof. Since $R$ is not nil, not all symmetric elements of $R$ are nilpotent (Proposition 1). Since $R$ satisfies (1), at least one symmetric element must be regular. Next we prove that each element $x$ of $R$ is regular iff $x$ is regular modulo $N$. Since $R$ is noetherian, the 'only if' part follows from [2]. To see the 'if' part one can proceed as follows. Clearly $s=x x^{*}$ is regular modulo $N$ but cannot be nilpotent. By (1), $s$ is then regular. Consequently $x$ is left regular. Similarly $x$ is right regular. By a result of Small [13], $R$ is a left order in an artinian ring with unity $Q$. As in [10], the involution * in $R$ extends to a unique involution in $Q$.

Henceforth we assume that $R$ is as in Proposition 2. Since in an artinian ring the nilpotent elements have bounded index, one gets

Proposition 3. The nilpotent symmetric elements of $R$ have a bounded index.

Proof. By Propositions 1 and 2.

PROPOSITION 4. The largest proper symmetric ideal of the ring of fractions $Q$ is precisely the Jacobson radical $\operatorname{Rad} Q$ of $Q$.

Proof. Let $J=J^{*} \neq Q$ be a symmetric ideal of $Q$. Let $J_{0}=J \cap R$. This is a symmetric ideal of $R$ which cannot contain regular elements. By (1), each symmetric element of $J_{0}$ is nilpotent. By Propositions 1 and $2, J_{0}$ is nil. Thus $J_{0} \subseteq N$. Consequently $J=J_{0} \cdot Q \subseteq N \cdot Q=\operatorname{Rad} Q$. Since the latter ideal is a proper symmetric ideal of $Q$, the proposition is established.

PROPOSITION 5. The factor ring $R_{1}=R / N$ is a ring satisfying (1), which is an order in a semisimple artinian ring $Q_{1}=Q / \operatorname{Rad} Q$ uith no symmetric ideals (except 0 and $Q_{1}$ ).

ProOf. Let $s_{1}$ be a symmetric element of $R_{1}$. Let $s$ be a symmetric element of $R$ which is mapped on $s_{1}^{2}$. If $s$ is nilpotent so must be $s_{1}$. If $s$ is regular, $s_{1}^{2}$ is regular (Goldie's result) and consequently $s_{1}$ is regular. Thus $R_{1}$ satisfies (1). The rest of Proposition 5 follows at once from Propositions 1 and 4.

Proposition 6. Any artinian ring $Q$ with unity admitting a left order $R$ satisfying (1) must satisfy (2).

Proof. Since $\operatorname{Rad} Q$ is nilpotent, $Q$ and $Q_{1}=Q / \operatorname{Rad} Q$ satisfy at the same time (2). This, in conjunction with the above proposition, enables us to assume that $N=\operatorname{Rad} Q=0$. Consequently $Q$ has no symmetric ideals. 
As is well known, $Q$ is then either a simple ring (I) or $Q$ is a direct sum of two ideals of the form $Q=I \oplus I^{*}$ (II).

Case (II). Let $I_{0}=I \cap R$. We have $I_{0} \cap I_{0}^{*}=0$. For each $x \in I_{0}$, set $s=$ $s(x)=x \oplus x^{*}=s^{*}$. By (1), either $s$ is nilpotent or regular. By the directness of the sum, $x$ is regular or nilpotent. Since $R$ is a noetherian ring which is semiprime, $I_{0}$ does not have nil ideals. From this $I_{0}$ is a skew-domain (see for example [7, Theorem 1]). Consequently $I$ is a division ring isomorphic to $Q / I^{*}$. Since $R / I_{0}^{*}$ is an order in $Q / I^{*}$, a division ring, $R / I_{0}^{*}$ is a skewdomain.

Case (I). By the lemma below, there are an integer $n$, a division ring $F$, an order $S$ in $F$, and a *-isomorphic copy $R^{\prime}$ of $R$ such that $S_{n} \leqq R^{\prime} \leqq F_{n} \approx Q$, where the involution in $R^{\prime}$ extends to an involution * in $F_{n}$ as in Lemma 1 (3) or (4). Suppose that the involution is as in Lemma 1(3). If $n$ were $>1$ consider the matrix $a e_{12}$ where $0 \neq a \in S$. We have $\alpha=a e_{12}\left(a e_{12}\right)^{*}=$ $a \gamma_{1}^{-1} a^{*} \gamma_{2} e_{11}$. Since $a \neq 0, a \gamma_{1}^{-1} a^{*} \gamma_{2} \neq 0$, then $\alpha$ is neither nilpotent nor invertible. However since $a e_{12} \in S_{n} \subseteq R^{\prime},\left(a e_{12}\right)^{*} \in R^{\prime}$. Consequently $\alpha=$ $\alpha^{*} \in R^{\prime}$, a contradiction. We must conclude that $Q \approx F$, a division ring. On the other hand, if the involution is as in Lemma 1(4) we have necessarily $n=2$. For in the opposite case take $0 \neq a \in S$. Let $a$ be the matrix of $F_{n}$ all of whose blocks are 0 except the upper left-hand block $=\left(\begin{array}{cc}a & 0 \\ 0 & a\end{array}\right)$. Again, $\alpha=a(a)^{*}$ is neither nilpotent nor invertible but $\alpha \in R^{\prime}$, a contradiction. We must conclude that $Q \approx F_{2}$ is the $2 \times 2$-matrices over the field $F$ with the symplectic involution $\left(\begin{array}{ll}a & b \\ c & d\end{array}\right) \rightarrow\left(\begin{array}{cc}d & -b \\ -c & a\end{array}\right)$, which obviously satisfies (2).

The following lemma is just a combination of two results due to Faith and Utumi and to Jacobson ([5, p. 272], [1, Remark 4]).

LeMma 1 (FAITH, UtUMI, JACOBSON). Let $R$ be a ring with involution which is an order in a right artinian simple ring $Q$. There are an integer $n, a$ division ring $F$, an order $S$ in $F$, and $a^{*}$-isomorphic copy $R^{\prime}$ of $R$ satisfying the following conditions (1) and (2), and one of the conditions (3) or (4):

(1) $S_{n} \leqq R^{\prime} \leqq F_{n} \approx Q\left(S_{n}\left(F_{n}\right)\right.$ the $n \times n$-matrices over $\left.S(F)\right)$.

(2) The involution in $R^{\prime}$ extends to an involution in $F_{n}$ leaving globally fixed the subdivision ring of scalar matrices.

(3) There is a system of matrix units $\left\{e_{i j}\right\}_{i j}$ and there is a sequence of nonzero elements $\gamma_{1}, \cdots, \gamma_{n} \in F$ which are symmetric elements under the induced involution in $F$ so that

for any $a_{i j} \in F$.

$$
\left(\sum_{i j} a_{i j} e_{i j}\right)^{*}=\sum_{j i} \gamma_{i}^{-1} a_{i j}^{*} \gamma_{j} e_{j i}
$$

(4) $n$ is even, $F$ is a field. The matrix $\left(a_{r t}\right)_{r t}$ can be partitioned into square blocks $\left(\begin{array}{cc}a & b \\ c & d\end{array}\right)$ of coordinates $i, j=1, \cdots, n / 2$. The $j \times i$-block in $\left[\left(a_{r t}\right)_{r t}\right]^{*}$ will be $\left(\begin{array}{cc}d & -b \\ -c & a\end{array}\right)$ where $\left(\begin{array}{ll}a & b \\ c & d\end{array}\right)$ is the $i \times j$-block in $\left(a_{r t}\right)_{r t}$. 
Combining Propositions 1 and 6 one gets at once a proof of the Theorem. In the course of the proof of Proposition 6 it was seen that

PROPOSITION 7. If $R$ is a semiprime noetherian ring satisfying (1) then $R$ is of one of the following types: (i) $R$ is a skew-domain; (ii) $R$ is a subdirect product of a sken-domain by itself; (iii) $R$ is in order in the $2 \times 2$-matrices over a field.

An obvious consequence of the above proposition is that each norm $x x^{*}$ and trace $x+x^{*}$ of the considered ring $R$ which is nilpotent must be 0 . This, in conjunction with Proposition 5, gives at once a proof of the Corollary. Let us conclude with the following.

REMARK. One could wonder if the information (i)-(iii) in the Corollary can be lifted to the ring $R$ itself. This is possible for (i) and (iii). In fact (i) is equivalent with Lesieur's condition that (i)' each element $x$ of $R$ is nilpotent or regular; (iii) is equivalent with the condition that $R$ is an order in the $2 \times 2$-matrices $Q$ over an artinian ring $L$ such that $L / \operatorname{Rad} L$ is a field [5, p. 56]. As for (ii), one would be tempted to think that it means that $R$ is a subdirect product of rings satisfying (i)' ${ }^{\prime}$. The following example shows that this is not generally true.

ExAmple 2. Let $F$ be any field. Let $R$ be the ring of 2-by-2 upper triangular matrices with entries from $F$. It can be verified that $R$ is a right artinian subdirectly irreducible ring with the involution $*:\left(\begin{array}{ll}a & b \\ 0 & c\end{array}\right) \rightarrow\left(\begin{array}{ll}c & -b \\ 0 & a\end{array}\right)$. Under * each symmetric element is nilpotent or invertible. Here $R / \operatorname{Rad} R=R / N \approx F \times F$ but since $R$ is subdirectly irreducible $R$ is not a subdirect product of the desired type.

\section{REFERENCES}

1. W. E. Baxter and W. S. Martindale III, Rings with involution and polynomial identities, Canad. J. Math. 20 (1968), 465-473. MR 36 \#5168.

2. W. A. Goldie, The transfer ideal, Séminaire d'algèbre non commutative, Orsay, 1967/1968, Conf. 1.

3. I. N. Herstein and S. Montgomery, Invertible and regular elements in rings with involution, J. Algebra (to appear).

4. I. N. Herstein, Topics in ring theory, Univ. of Chicago Press, Chicago, Ill., 1969. MR 42 \#6018.

5. N. Jacobson, Structure of rings, rev. ed., Amer. Math. Soc. Colloq. Publ., vol. 37, Amer. Math. Soc., Providence, R.I., 1964. MR 36 \#5158.

6. C. Lanski, Rings with involution whose symmetric elements are regular, Proc. Amer. Math. Soc. (to appear).

7. L. Lesieur, Anneaux noetheriens à gauche completement primaires et anneaux coirréductibles, J. Reine Angew. Math. 241 (1970), 106-117. MR 41 \#3515.

8. K. McCrimmon, On Herstein's theorems relating Jordan and associative algebras, J. Algebra 13 (1969), 382-392. MR 40 \#2721. 
9. K. McCrimmon, Quadratic Jordan algebras whose elements are all invertible or nilpotent, Proc. Amer. Math. Soc. 35 (1972), 309-316.

10. S. Montgomery, Polynomial identity algebras with involution, Proc. Amer. Math. Soc. 27 (1971), 53-56. MR 42 \#4590.

11. J. M. Osborn, Jordan algebras of capacity two, Proc. Nat. Acad. Sci. U.S.A. 57 (1967), 582-588. MR 35 \#6727.

12. - Jordan and associative rings with nilpotent and invertible elements, $\mathrm{J}$. Algebra 15 (1970), 301-308. MR 41 \#6925.

13. L. Small, Orders in Artinian rings, J. Algebra 4 (1966), 13-41. MR 34 \#199.

Département de Mathématique, Université D'Amiens, Amiens, France

Department of Mathematics, Carleton University, Ottawa, Ontario, Canada 